\title{
Sensitivity analysis on the model input parameters of flapping wing kinematics for optimum level flight via particle swarming optimization
}

\author{
Mohd Faisal Abdul Hamid \\ Department of Aerospace Engineering, Faculty of Engineering, Universiti Putra Malaysia, 43400 Serdang, Selangor, Malaysia. \\ *Corresponding author E-mail: mohd_faisal@upm.edu.my
}

\begin{abstract}
A systematic reviewing process to assess the sensitivity of the input parameters of flapping wing kinematics for optimum level flight is presented. This is done prior to the development of a stroke optimization model to predict the aerodynamic performance of an insect (hawk moth) during flight. A systematic iterative process-population-based stochastic algorithm, known as particle swarming optimization, is used. In the search for an optimal realistic wing kinematic motion, several constraints for stable flight are defined following the observational wing kinematics data from experiments on real insects. This is to avoid any physically-unrealistic solutions of the wing motion. Two stages of sensitivity analyses are conducted via partial sensitivity analysis, or one-at-a-time. First, sensitivity screening analyses are performed to gauge the dependability of the solution output, i.e. total force and total power, on each model input parameter; a total of 9 model input parameters. Then, the second stage of sensitivity analysis to measure on how the most sensitive model input parameters will affect the optimized kinematics are conducted. The results have shown that the wing length and the wing translational lift coefficient are the most sensitive aerodynamic model input parameters among other inputs.
\end{abstract}

Keywords: flapping wing; kinematic; PSO; optimal flapping motion; level flight.

\section{Introduction}

Over the past decade, numerous studies have been conducted to optimize the flight performance of the flapping wing. These mathematical optimization studies have been directed to a wide range of analyses, focusing on various aspects of optimization such as wing-body dynamics [1], wing shape [2,3], wing structural aeroelasticity [4], flight stability [5], and wing kinematics [6-12]. Following assessment on some of the flight performance optimization of flapping wing studies [5, 9], genetic algorithms and gradientbased optimizer are among those regularly used in the study of the flapping insect flight. In the meantime, several other studies opt to combine multiple optimization methods in the search of the optimal solution $[3,7]$.

Crucially, replicating the wing motion of an insect in flight would involve complex three-dimensional motion. Thus, an optimization procedure is required, and for this purposed, a systematic iterative process-population-based stochastic algorithm known as Particle Swarming Optimization [13] (PSO) has been adapted. This process has proved to be useful in assisting in the search for an optimal realistic wing kinematic motion $[7,14,15]$, which is subjected with several constraints for stable flight. As noted by Chen et al. [16], when compared to the classical optimization methods, PSO is reportedly much simpler to implement, more efficient for computation and has become one of the most popular optimization techniques for solving continuous optimization problems. Moreover, this method does not require assumptions about the problem being optimized and does not require that optimization problem to be differentiable, which is advantageous for the optimization prob- lems with very large spaces of candidate solutions that are partially irregular, noisy and time dependent [17].

Prior to the search of the kinematics of flapping wing model for optimum level flight, a systematic reviewing process to assess the uncertainty of the model input parameters is carried out via partial sensitivity analysis or one-at-a-time [18]. This is done to screen out the most sensitive parameters and see how they reflect on the optimized kinematics. The specified ranges for the optimization of the kinematic variables are defined following observational wing kinematics data from the experiments on real insects. This will give limits to all kinematic parameters and prevent any physicallyunrealistic solutions of the wing motion.

\section{Methodology}

\subsection{Cost function and constraints}

The cost function of the optimization is the propulsive efficiency; with constraints on the flight stability, muscular power ratio, flight muscle ratio and wing kinematics. This optimization process is carried out iteratively, with stopping criterion of up to 1,000 iterations and $10^{-4}$ tolerance, whichever that comes first. The specified range for the optimal model parameters are shown in Table 1 .

The upper bound value of $C_{\eta}$ is set to be at a maximum of 5 [20]. This value is based on some studies of the real insects' flight [2123 ] and is used here to avoid an unrealistically-high rate of wing rotation. Additionally, to comply with the physical power of the real insect, the available power must be limited [24]. Following Ref. [25-28], the maximum flight muscle ratio $\mathrm{m}_{\text {muscle }} / \mathrm{m}_{\text {insect }}$ 
and the available muscular power ratio $\mathrm{P} / \mathrm{m}_{\text {muscle }}$ can be assumed to be equal to $60 \%$ and $150 \mathrm{~W} / \mathrm{kg}$, respectively. Hence, by equating these two values, the available power $\mathrm{P} / \mathrm{m}_{\text {insect }}$ is estimated to be a maximum of $90 \mathrm{~W} / \mathrm{kg}$.

Table 1: Range (or constraint) for optimal model parameters [19]

\begin{tabular}{|c|c|c|}
\hline \multirow{2}{*}{ Parameter } & \multicolumn{2}{|c|}{$V_{\infty}=\mathbf{5 . 0 0} \mathbf{~ m} / \mathbf{s}$} \\
\cline { 2 - 3 } & Lower limit & Upper Limit \\
\hline$f$ & $49.20^{0}$ & $24.80 \mathrm{~Hz}$ \\
\hline$\beta$ & $49.40^{0}$ & $53.20^{0}$ \\
\hline$\phi_{m}$ & $1.0^{0}$ & $20.45^{0}$ \\
\hline$\theta_{m}$ & $1.0^{0}$ & $90.0^{0}$ \\
\hline$\eta_{m}$ & $-30.0^{0}$ & $30.0^{0}$ \\
\hline$\phi_{0}$ & $-30.0^{0}$ & $30.0^{0}$ \\
\hline$\theta_{0}$ & $-90.0^{0}$ & $90.0^{0}$ \\
\hline$\eta_{0}$ & $-180.0^{0}$ & $180.0^{0}$ \\
\hline$\Phi_{\phi}$ & $-180.0^{0}$ & $180.0^{0}$ \\
\hline$\Phi_{\theta}$ & 0.01 & 1.00 \\
\hline$\Phi_{\eta}$ & $0.0^{0}$ \\
\hline$K$ & \multicolumn{2}{|c|}{1 or 2} \\
\hline$N$ & \multicolumn{2}{|c|}{} \\
\hline$C_{\eta}$ & \multicolumn{2}{|c|}{} \\
\hline \multicolumn{2}{|c|}{} \\
\hline
\end{tabular}

\subsection{Working procedure}

In the optimization process, a group of particles is selected (e.g. 10 particles for each optimized parameter), with the particles searching or swarming towards the desired solution simultaneously within a constrained-solution space. Each of the individual particles has a bidirectional link with its neighbours, and is assigned to communicate along the process. The communication and interactions take place in a way that individual particles will reconfigure their current position at each time step, by adding their velocity to their previous position. The procedure can be represented by the following Eqn. 1 and Eqn. 2, where $j$ is the target particle's index; $d$ is the particle dimension; $x_{j}$ is the particle's position; $v_{j}$ is the velocity; $p_{j}$ is the best position found so far by $j ; q$ is the index of $j$ 's best neighbour; $U\left(0, \beta_{p s o}\right)$ is a uniform random number generator; $\alpha_{p s o}$ is the inertia weight or constriction coefficient and $\beta_{p s o}=\psi_{p s o} / 2$ is the acceleration constant [29].

$$
\begin{aligned}
v_{j d}^{(t+1)} \leftarrow \alpha_{p s o} v_{j d}^{(t)} & +U\left(0, \beta_{p s o}\right)\left(p_{j d}-x_{j d}^{(t)}\right) \\
& +U\left(0, \beta_{p s o}\right)\left(p_{q d}-x_{j d}^{(t)}\right)
\end{aligned}
$$

The program systematically evaluates each single parameter vector of particle $j$ in the functions $\left(x_{j d}^{(t+1)}\right.$ and $\left.v_{j d}^{(t+1)}\right)$, and compares the result to the best result obtained by $j$ thus far. Each particle cycles around a region centred on the centroid of the previous best particle's position and with the best neighbours. If the current result is the best so far, the best position is updated with the current position, and the previous best function result is updated with the current result. As these variables are updated, each particle trajectory shifts to a new region, closer to the optima of the search space until the desired results from the improved function are obtained. This is shown in Figure 1.

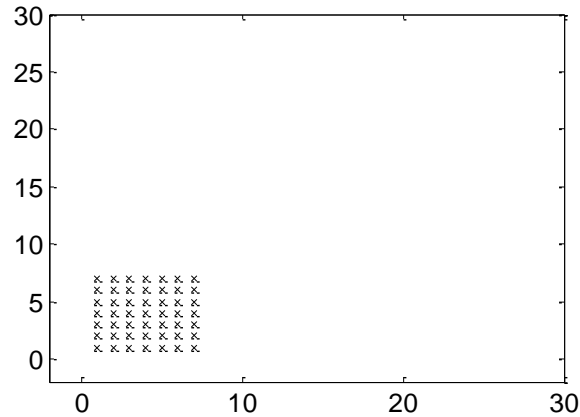

(a)

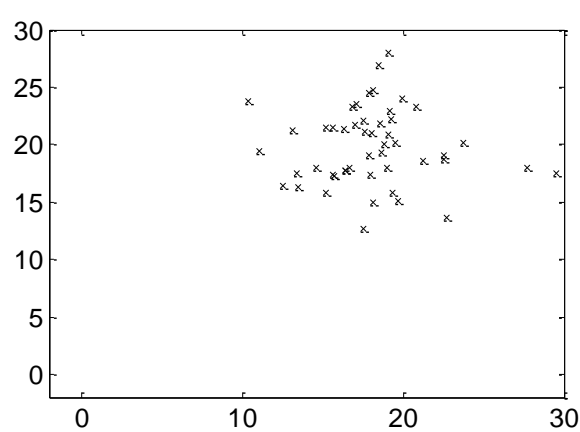

(b)

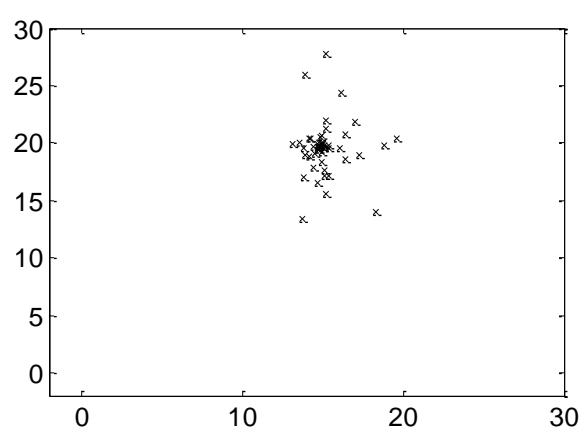

(c)

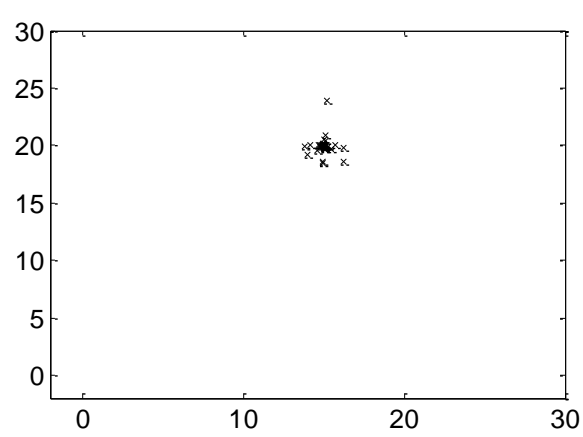

(d)

Fig. 1: Sample of particle swarming movement distribution from PSO analysis; (a) at $1^{\text {st }}$ iteration; (b) at $5^{\text {th }}$ iterations; (c) at $10^{\text {th }}$ iterations; (d) at $20^{\text {th }}$ iterations. 


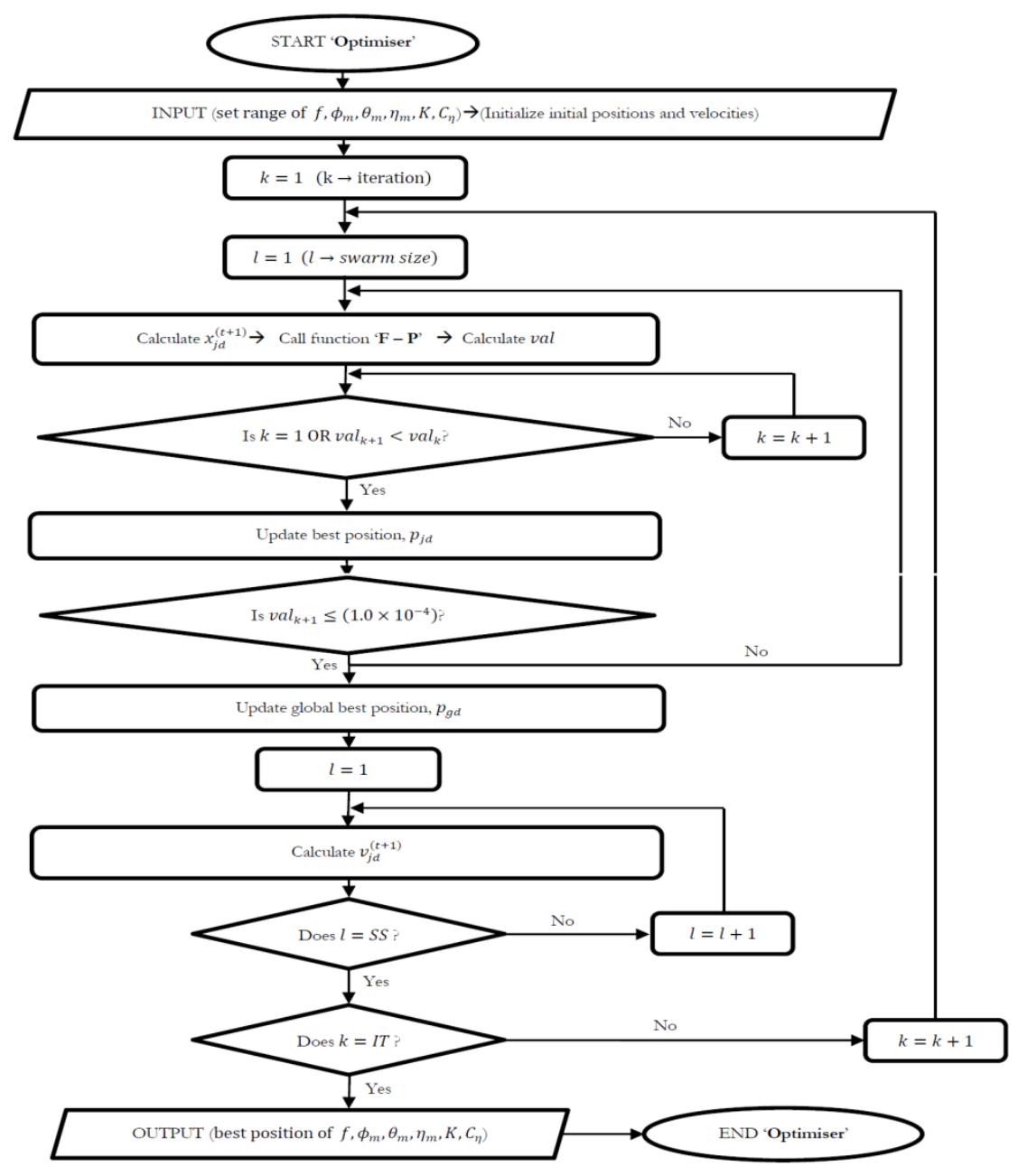

Fig. 2: Flow structure for the optimization program

\subsection{Sensitivity analysis}

Sensitivity analysis is a systematic reviewing process to assess the uncertainty of a model. In this study, two sensitivity analyses are conducted via partial sensitivity analysis or one-at-a-time [18]. Firstly, the sensitiveness of the aerodynamic model input parameters on the results (total force and power) is assessed. Secondly, having determined the sensitive model input parameters, the variations in optimized kinematics are assessed. Each of the analyses is performed by changing the value of one parameter from its baseline values (Table 2) while maintaining the others as a constant, and evaluating its effect on the results at that time.

Table 2: Model input parameters for sensitivity analysis

\begin{tabular}{|c|c|c|}
\hline Wing length & Wing mean chord & Wing mass \\
\hline$R$ & $\bar{c}$ & $m_{\text {wing }}$ \\
\hline $51.9 \mathrm{~mm}$ & $18.26 \mathrm{~mm}$ & $47 \mathrm{mg}$ \\
\hline $\begin{array}{c}\text { Translational lift } \\
\text { coefficient }\end{array}$ & $\begin{array}{c}\text { Drag coefficient at } \\
\boldsymbol{\alpha}=\mathbf{0}^{\mathbf{0}}\end{array}$ & $\begin{array}{c}\text { Drag coefficient at } \\
\boldsymbol{\alpha}=\mathbf{9 0}^{\mathbf{0}}\end{array}$ \\
\hline$C_{t}$ & $C_{D}(0)$ & $C_{D}(\pi / 2)$ \\
\hline 1.631 & 0.07 & 3.06 \\
\hline $\begin{array}{c}\text { Rotational lift coeffi- } \\
\text { cient }\end{array}$ & $\begin{array}{c}\text { Non-dimensional } \\
\text { viscous torque }\end{array}$ & $\begin{array}{c}\text { Non-dimensional } \\
\text { viscous torque }\end{array}$ \\
\hline$C_{r}$ & $\mu_{1}$ & $\mu_{2}$ \\
\hline$\pi$ & 0.2 & 0.2 \\
\hline
\end{tabular}

This approach allows the gauging of the dependability of the solution output on each input parameter and helps in determining the key parameters that most significantly affect the results. In addition, it would be useful in indicating the consistency of the optimized output kinematics upon the changes of the determined sen- sitive model input parameters. Flow structure for the optimization program is shown in Figure 2.

\section{Results and discussions}

The sensitivity analysis of the model input parameters on the results is performed using defined wing kinematics for optimum level flight of the hawk moth at velocity, $V=5 \mathrm{~m} / \mathrm{s}$ (the wing kinematics are given in Table 1).

\subsection{Sensitivity screening analysis}

As shown in Figure 3, the result indicates that the changes of wing length and wing translational lift coefficient have the greatest influence on both the total force $\boldsymbol{F}$ and power $\boldsymbol{P}$. This result is logical, since these are the key drivers allied with the total area of the wing aerodynamic surface $(\boldsymbol{R})$, and the aerodynamic efficiency $\left(\boldsymbol{C}_{\boldsymbol{t}}\right)$ of the wing. A larger wing area (or translational lift coefficient) will translate into generating a greater amount of force, and hence increasing the power required $(\boldsymbol{P}=\boldsymbol{F V})$. 


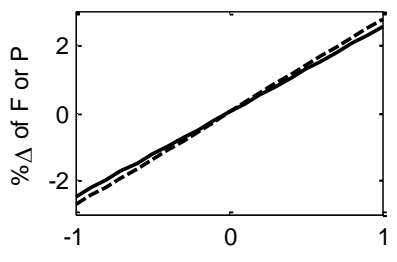

$\mathrm{C}_{\mathrm{t}}$

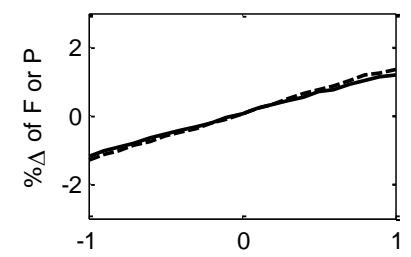

$\mathrm{C}_{\mathrm{r}}$

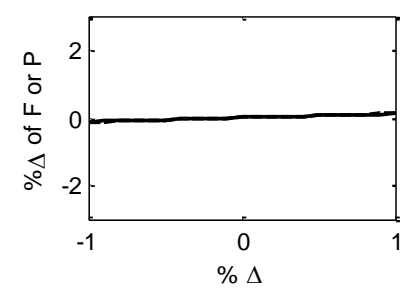

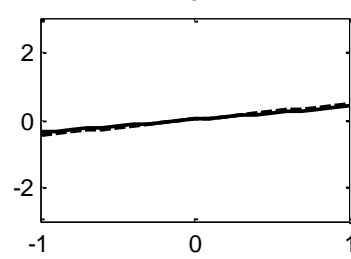

$C_{d}(0)$

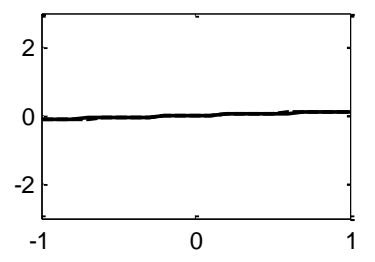

$\mu_{1}$

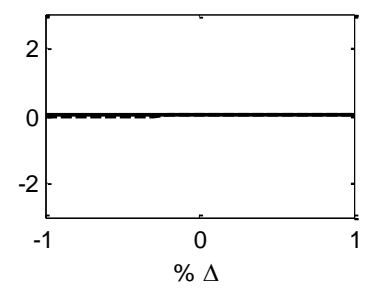

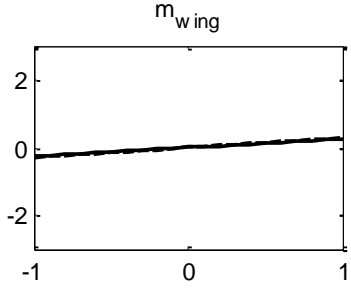

$C_{d}(\pi / 2)$

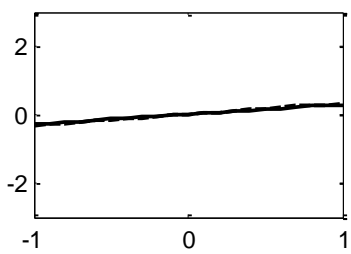

$\mu_{2}$

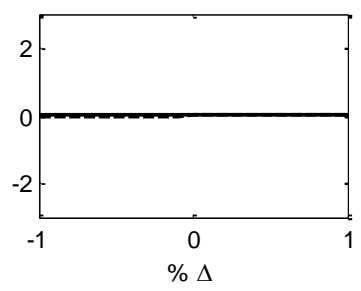

Fig. 3: Sensitivity screening analysis, showing the variation in total force $\boldsymbol{F}$ (solid line) and power $\boldsymbol{P}$ (dashed line). $\boldsymbol{F}$ and $\boldsymbol{P}$ are shown as percentage of the baseline values from Table 2 .

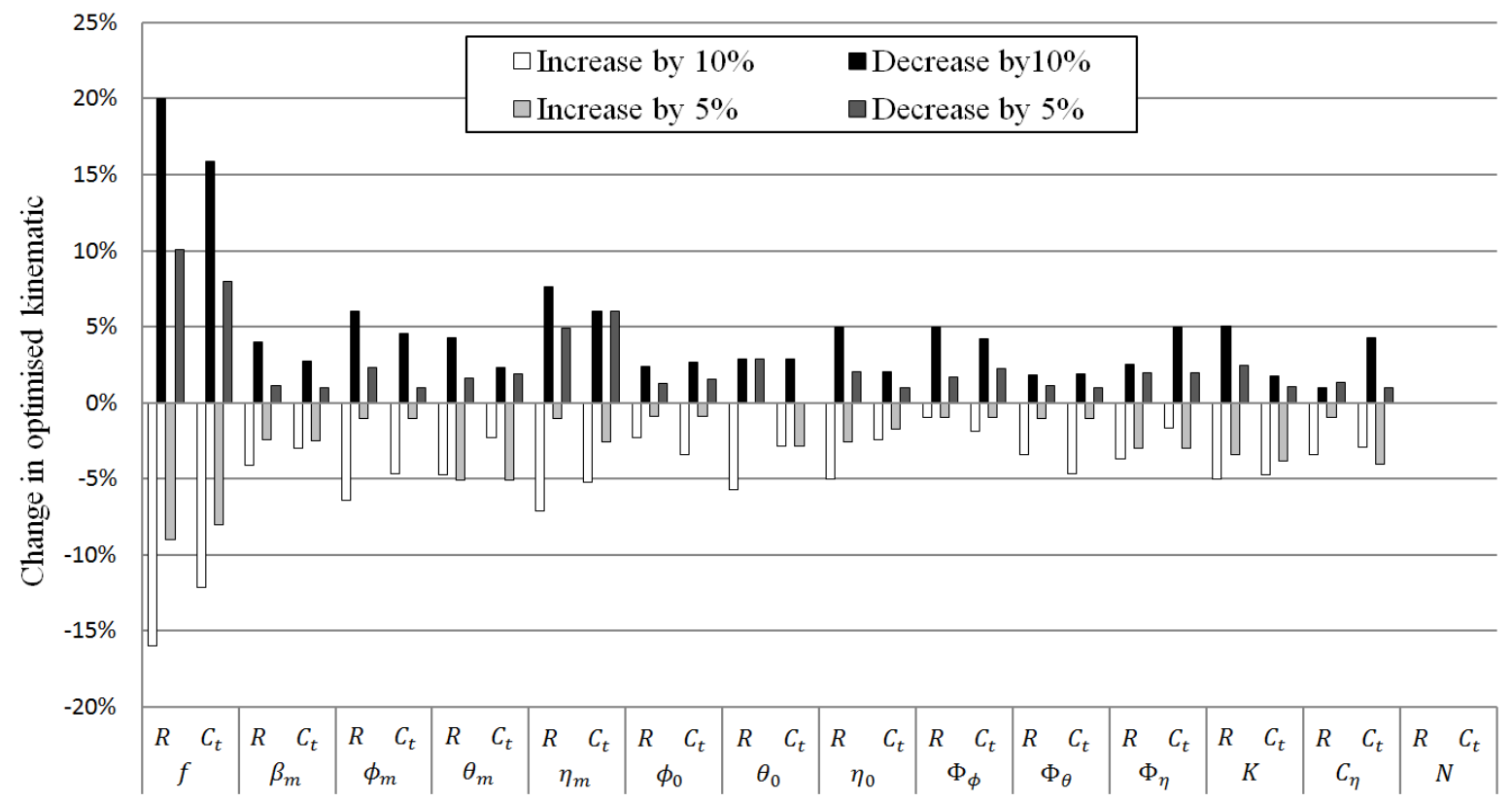

Fig. 4: Variations in optimized kinematics following the changes in each of the screened model input parameter $\left(\boldsymbol{R}, \boldsymbol{C}_{\boldsymbol{t}}\right)$. Changes in optimized kinematics are shown as percentages of the baseline values from level flight of the hawk moth at $V=5$ m/s

\subsection{Sensitivity variations in the optimized kinematics}

From the sensitivity screening analysis of the model input parameters, it is shown that the wing length and the wing translational lift coefficient are the most sensitive among other inputs. For the next part, the variations in optimized kinematics are measured by performing another sensitivity analysis, following the changes in the screened model input parameters $\left(\boldsymbol{R}, \boldsymbol{C}_{\boldsymbol{t}}\right)$. This analysis is intended to measure how the model input parameters will affect the optimised kinematics. In this analysis, the model parameters are increased (or decreased) by 5\% and 10\% from base value as given in Table 2; this provides an ample range for measuring the variations in the optimized kinematics.
Figure 4 shows that an increase in model input parameter $\left(\boldsymbol{R}, \boldsymbol{C}_{\boldsymbol{t}}\right)$ reduces the changes in the optimised kinematics, and vice-versa. Overall, the changes in the wing length delivered a greater impact on the optimised model input parameters than the wing translational lift coefficient. The changes in the model input parameters had a noticeable influence on the optimized frequency of the wing. Increasing the value of the model input parameters reducing the power required by up to $\sim 30 \%$. This is because an increase in the wing size produces wings with a bigger aerodynamic surface area that will benefit from lower flapping frequencies and hence reducing the power required [15]. 


\section{Conclusions}

Two stages of sensitivity analyses have been carried out, involving a total of 9 model input parameters. In the first stage, a sensitivity screening analysis is performed to gauge the dependability of the solution output on each model input parameter. This is followed by the second stage of sensitivity analysis to measure on how the most sensitive model input parameters will affect the optimised kinematics. By applying these two stages of sensitivity analyses approach, it would helps in determining the key parameters that most significantly affecting the results. As shown from the sensitivity screening analysis, the wing length and the wing translational lift coefficient are the most sensitive aerodynamic model input parameters among other inputs. This is due to the fact that a larger wing surface area and a larger value of translational lift coefficient will produce a much greater amount of force and correspondingly, increasing the amount of power required. From the second stage of the sensitivity analysis, inverse solution/relationship has been identified. By increasing the value of both of the sensitive model input parameters, i.e. wing length and wing translational lift coefficient, the changes in all optimized kinematics are reduced. On the contrary, when both of them are reduced, all optimized kinematics are increased. Overall, the results obtained from this twostage sensitivity analyses are screened to be consistent. This finding is useful as it will help to understand and correlate those assigned parameters with the physical nature of flapping wing.

\section{Acknowledgement}

The author would like to thank the Universiti Putra Malaysia for providing the financial support through the Geran Putra IPM, Grant Agreements GP-IPM/2017/9537900. Also, the author would like to acknowledge Dr. Antonino Filippone (University of Manchester) for his contributions in the early stage of the work.

\section{References}

[1] Stanford B, Beran P \& Patil M (2013), Optimal flapping-wing vehicle dynamics via Floquet multiplier sensitivities. Journal of Guidance, Control, and Dynamics 36, 454-466.

[2] Ghommem M et al. (2012), Global optimization of actively morphing flapping wings. Journal of Fluids and Structures 33, 210-228.

[3] Ghommem M, Collier N, Niemi AH \& Calo VM (2014) On the shape optimization of flapping wings and their performance analysis. Aerospace Science and Technology 32, 274-292.

[4] Isogai K \& Harino Y (2007), Optimum aeorelastic design of a flapping wing. AIAA Journal 44, 2040-2048.

[5] Stanford B, Beran P, Snyder R \& Patil M (2013), Stability and power optimality in time-periodic flapping wing structures. Journal of Fluids and Structures 38, 238-254.

[6] Taha HE, Hajj MR \& Nayfeh AH (2013), Wing kinematics optimization for hovering micro air vehicles using calculus of variation. Journal of Aircraft 50, 610-614.

[7] Berman GJ \& Wang ZJ (2007), Energy-minimizing kinematics in hovering insect flight. Journal of Fluid Mechanics 582, 153-168.

[8] Khan ZA \& Agrawal SK (2011), Optimal hovering kinematics of flapping wings for micro air vehicles. AIAA Journal 49, 257-268.

[9] Tuncer IH \& Kaya M (2005), Optimization of flapping airfoils for maximum thrust and propulsive efficiency. AIAA journal 43, 23292336.

[10] Zheng L, Hedrick T \& Mittal R (2013), A multi-fidelity modelling approach for evaluation and optimization of wing stroke aerodynamics in flapping flight. Journal of Fluid Mechanics 721, 118-154.

[11] Soueid H, Guglielmini L, Airiau C \& Bottaro A (2009), Optimization of the motion of a flapping airfoil using sensitivity functions. Computers \& Fluids 38, 861-874.

[12] Kaya M, Tuncer IH, Jones KD \& Platzer MF (2009), Optimization of flapping motion parameters for two airfoils in a biplane configuration. Journal of Aircraft 46, 583-592.

[13] Kennedy J (2010), Particle swarm optimization. Encyclopedia of Machine Learning, 760-766.
[14] Faisal AHM \& Filippone A (2016), Aerodynamic model for insect flapping wings with induced flow effect. Journal of Aircraft 53(3), 701-712.

[15] Faisal AHM \& Filippone A (2016), Aerodynamic model for tandem flapping wings. AIAA Journal 54(12), 3849-3858.

[16] Chen WN et al. (2010), A novel set-based particle swarm optimization method for discrete optimization problems. IEEE Transactions on Evolutionary Computation 14, 278-300.

[17] Pontani M \& Conway BA (2010), Particle swarm optimization applied to space trajectories. Journal of Guidance, Control, and Dynamics 33.

[18] Saltelli A, Chan K \& Scott EM (2000), Sensitivity analysis. Wiley.

[19] Willmott AP \& Ellington CP (1997), The mechanics of flight in the hawk moth Manduca sexta. I. Kinematics of hovering and forward flight. The Journal of Experimental Biology 200, 2705-2722.

[20] Nabawy MR \& Crowther WJ (2015), Aero-optimum hovering kinematics. Bioinspiration \& Biomimetics Journal 10, 1-10.

[21] Sun M \& Du G (2003), Lift and power requirements of hovering insect flight. Acta Mechanica Sinica 19, 458-469.

[22] Liu Y \& Sun M (2008), Wing kinematics measurement and aerodynamics of hovering droneflies. Journal of Experimental Biology 211, 2014-2025.

[23] Altshuler DL, Dickson WB, Vance JT, Roberts SP \& Dickinson MH (2005), Short-amplitude high-frequency wing strokes determine the aerodynamics of honeybee flight. Proceedings of the $\mathrm{Na}$ tional Academy of Sciences of the United States of America 102, 18213-18218.

[24] Stanford B, Kurdi M, Beran P \& McClung A (2012), Shape, structure, and kinematic parameterization of a power-optimal hovering wing. Journal of Aircraft 49, 1687-1699.

[25] Willmott AP \& Ellington CP (1997), The mechanics of flight in the hawk moth Manduca sexta. II. Aerodynamic consequences of kinematic and morphological variation. Journal of Experimental Biology 200, 2723-2745.

[26] Wakeling JM \& Ellington CP (1997), Dragonfly Flight III: Lift and power requirements. The Journal of Experimental Biology 200, 583-600.

[27] Marden JH (1989), Bodybuilding dragonflies: costs and benefits of maximizing flight muscle. Physiological Zoology 62, 505-521.

[28] Willmott AP (2005), Mechanics of hawk moth flight. Cambridge University.

[29] Clerc M \& Kennedy J (2002), The particle swarm-explosion, stability, and convergence in a multidimensional complex space. IEEE Trans. on Evolutionary Computation 6, 58-73. 